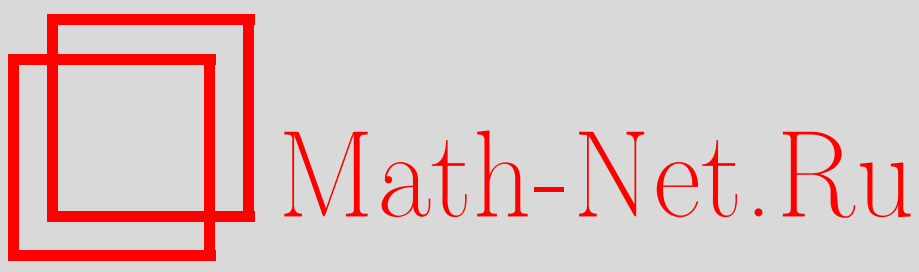

А. И. Булгаков, В. В. Скоморохов, Аппроксимация дифференциальных включений, Матем. сб., 2002, том 193, номер 2, 35-52

DOI: https://doi.org/10.4213/sm626

Использование Общероссийского математического портала Math-Net.Ru подразумевает, что вы прочитали и согласны с пользовательским соглашением http://www.mathnet.ru/rus/agreement

Параметры загрузки:

IP: 3.85 .183 .62

26 апреля 2023 г., $18: 26: 36$ 
УДК 517.911

\author{
А.И. Булгаков, В.В. Скоморохов
}

\title{
Аппроксимация дифференциальных включений
}

\begin{abstract}
В работе рассматриваются методы аптроксимации диффференциального включения, правая часть которого удовлетворяет условиям Каратеодори. Получены необходимые и достаточные условия того, что аптроксимация дифференциалннго включения является устойчивой относительно внутренних и внешних возмущений, т.е. "небольшие" изменения (в смысле расстояния по Хаусдорфу) правой части включения приводят к "небольшому" изменению множества решений.

Библиография: 20 названий.
\end{abstract}

Понятие приближенного решения ( $\delta$-решения) дифференциального включения введено А.Ф. Филипповым (см. [1]). Это определение имеет важное значение для изучения дифференциальных включений с выпуклозначной и полунепрерывной сверху правой частью, поскольку пределы сходяшихся последовательностей приближенных решений являются решениями (см. [1]). В настоящей работе для изучения аппроксимаций дифференциальных включений рассматривается несколько другое определение понятия приближенного решения. Отличие сформулированного здесь решения от приближенного решения по Филиппову заключается в том, что значения многозначных отображений, определяющие "приближенные дифференциальные включения", не "овыпукляются". Как оказалось, такое определение приближенного решения полезно для исследования аппроксимаций дифференциальных включений с невьпуклой правой частью. Оно, прежде всего, позволяет доказать, что аппроксимация включений с невыпуклой правой частью может быть не устойчивой операцией, поскольку "небольшие" изменения правой части могут привести к существенному изменению множеств приближенных решений. Кроме того, это определение позволяет получить необходимое и достаточное условие того, что аппроксимация является устойчивой. В работе это условие носит название принципа плотности. Отметим также, что здесь исследование проводится на основе свойств квазирешений дифференциальных включений. Понятие квазирешения (квазитраектории) включения дано Важевским (T. Wazewski, cм. [2], [1]).

Пусть $\mathbb{R}^{n}$ - пространство $n$-мерных вектор-столбцов с нормой $|\cdot| ; \operatorname{comp}\left[\mathbb{R}^{n}\right]$ - множество всех непустых ограниченных замкнутых подмножеств пространства $\mathbb{R}^{n} ; B[u, r]$ - замкнутый шар пространства $\mathbb{R}^{n}$ с центром в точке $u$ и радиусом $r>0 ; B[u, 0] \equiv\{u\}$. Пусть $V \subset \mathbb{R}^{n}$. Обозначим через $\bar{V}$ замькание множества $V$, через со $V$ - выпуклую оболочку множества $V ; V^{\varepsilon} \equiv \overline{\bigcup_{u \in V} B[u, \varepsilon]}$, если $\varepsilon>0$, и $V^{0} \equiv \bar{V} ;\|V\|=\sup \{|u|: u \in V\}$.

Работа выполнена при поддержке Российского фонда фундаментальных исследований (грант № 01-01-00140). 
Пусть $h^{+}\left[F_{1} ; F_{2}\right] \equiv \sup \left\{\rho\left[y, F_{2}\right]: y \in F_{1}\right\}$, где $\rho[\cdot, \cdot]-$ расстояние между точкой и множеством в пространстве $\mathbb{R}^{n}, h\left[F_{1}, F_{2}\right]=\max \left\{h^{+}\left[F_{1} ; F_{2}\right], h^{+}\left[F_{2} ; F_{1}\right]\right\}$ - хаусдорфово расстояние между множествами $F_{1}$ и $F_{2}$, содержашимися в пространстве $\mathbb{R}^{n}$.

Обозначим через $C^{n}[a, b]$ пространство непрерывных функций $x:[a, b] \rightarrow \mathbb{R}^{n} \mathrm{c}$ нормой $\|x\|_{C}=\max \{|x(t)|: t \in[a, b]\} ; L^{n}[a, b]-$ пространство суммируемых по Лебегу функций $x:[a, b] \rightarrow \mathbb{R}^{n}$ с нормой $\|x\|_{L}=\int_{a}^{b}|x(s)| d s$.

Непрерывность многозначных отображений понимается по Хаусдорфу. Измеримость однозначных функций понимаем по Лебегу (см. [3]), измеримость многозначных отображений понимаем в смысле [4].

Будем говорить, что отображение $F:[a, b] \times \mathbb{R}^{n} \rightarrow \operatorname{comp}\left[\mathbb{R}^{n}\right]$ удовлетворяет условиям Каратеодори, если выполняются следующие условия:

a) при каждом $x \in \mathbb{R}^{n}$ отображение $F(\cdot, x)$ измеримо;

b) при почти всех $t \in[a, b]$ отображение $F(t, \cdot)$ непрерывно;

c) для каждого ограниченного множества $V \subset \mathbb{R}^{n}$ найдется такая функция $\beta_{V}(\cdot) \in L^{1}[a, b]$, что при почти всех $t \in[a, b]$ и всех $x \in V$ выполняется неравенство $\|F(t, x)\| \leqslant \beta_{V}(t)$.

Обозначим через $S(F(\cdot))$ множество всех измеримых селекторов (ветвей) отображения $F:[a, b] \rightarrow \operatorname{comp}\left[\mathbb{R}^{n}\right]$. Отображение со $F:[a, b] \rightarrow \operatorname{comp}\left[\mathbb{R}^{n}\right]$ определим равенством $(\operatorname{co} F)(t)=\operatorname{co} F(t)$.

Обозначим через $K\left([a, b] \times \mathbb{R}^{n} \times[0, \infty)\right)$ множество всех функций $\eta:[a, b] \times \mathbb{R}^{n} \times$ $[0, \infty) \rightarrow[0, \infty)$, обладаюших следующими свойствами: при каждых $(x, \delta) \in \mathbb{R}^{n} \times$ $[0, \infty)$ функция $\eta(\cdot, x, \delta)$ измерима; при почти всех $t \in[a, b]$ и всех $\delta \in[0, \infty)$ функция $\eta(t, \cdot, \delta)$ непрерывна; для каждых $U \in \operatorname{comp}\left[\mathbb{R}^{n}\right]$ и $\delta \in[0, \infty)$ существует такая суммируемая функция $m_{U, \delta}:[a, b] \rightarrow[0, \infty)$, что при почти всех $t \in[a, b]$ и всех $x \in U$ и $\tau \in[0, \delta]$ выполняется неравенство $\eta(t, x, \tau) \leqslant m_{U, \delta}(t) ;$ при почти всех $t \in[a, b]$ и каждого $x \in \mathbb{R}^{n}$ выполняются равенства $\lim _{\delta \rightarrow 0+0}^{z \rightarrow x} \eta(t, z, \delta)=0$, $\eta(t, x, 0)=0$. Если для каждого $U \in \operatorname{comp}\left[\mathbb{R}^{n}\right]$ и $\delta \in[0, \infty)$ найдется такая функция $m_{U, \delta}(\cdot)$, определяющая множество $K\left([a, b] \times \mathbb{R}^{n} \times[0, \infty)\right)$, что она представляет собой константу, то множество таких функций $\eta(\cdot, \cdot, \cdot) \in K\left([a, b] \times \mathbb{R}^{n} \times[0, \infty)\right)$ обозначим через $\widetilde{K}\left([a, b] \times \mathbb{R}^{n} \times[0, \infty)\right)$.

Обозначим через $P\left([a, b] \times \mathbb{R}^{n} \times[0, \infty)\right)$ множество всех функций $\eta:[a, b] \times \mathbb{R}^{n} \times$ $[0, \infty) \rightarrow[0, \infty)$, обладающих свойствами из класса функций $K\left([a, b] \times \mathbb{R}^{n} \times[0, \infty)\right)$, а также удовлетворяющих следующим условиям: для каждых $U \in \operatorname{comp}\left[\mathbb{R}^{n}\right]$ и $\delta \in(0, \infty)$ найдутся такие числа $r(U, \delta)>0$ и $\beta(U, \delta) \geqslant 0$, что при почти всех $t \in[a, b]$, всех $x \in U$ число $r(U, \delta)$ удовлетворяет неравенству $r(U, \delta) \leqslant \eta(t, x, \delta)$, а для числа $\beta(U, \delta)$ при почти всех $t \in[a, b]$, всех $x \in U$ и $\tau \in[0, \delta]$ имеет место оценка $\eta(t, x, \tau) \leqslant \beta(U, \delta)$.

\section{§1. Аппроксимация с внешними возмущениями}

Рассмотрим дифференциальное включение

$$
\dot{x}(t) \in F(t, x(t)), \quad t \in[a, b],
$$

где отображение $F:[a, b] \times \mathbb{R}^{n} \rightarrow \operatorname{comp}\left[\mathbb{R}^{n}\right]$ удовлетворяет условиям Каратеодори. 
Под решением включения (1) будем понимать абсолютно непрерывную функцию $x:[a, b] \rightarrow \mathbb{R}^{n}$, удовлетворяющую этому включению при почти всех $t \in[a, b]$.

ОПРЕДЕЛЕНИЕ. Абсолютно непрерывная функция $x:[a, b] \rightarrow \mathbb{R}^{n}$ является квазирешением включения (1), если найдется такая последовательность абсолютно непрерывных функций $x_{i}:[a, b] \rightarrow \mathbb{R}^{n}, i=1,2, \ldots$, обладающая свойством: $x_{i}(\cdot) \rightarrow x(\cdot)$ в $C^{n}[a, b]$ при $i \rightarrow \infty$; для любого $i=1,2, \ldots$ и при почти всех $t \in[a, b]$ выполняется включение

$$
\dot{x}_{i}(t) \in F(t, x(t))
$$

Далее, будем считать, что если $x(\cdot)$ - квазирешение и $x(\cdot) \in V \subset C^{n}[a, b]$, то найдется такая последовательность абсолютно непрерывных функций $x_{i}(\cdot)$, $i=1,2, \ldots$, обладаюшая свойством из определения квазирешения включения (1), что для любого $i=1,2, \ldots$ выполняется включение $x_{i}(\cdot) \in V$.

ЗАмЕчАниЕ 1. Отметим, что определение квазирешения включения (1), сформулированное вьше, несколько отличается от определения квазитраектории по Важевскому (см. [1], [2], [5]) наличием условия (2). Понятия квазирешения включения (1) и квазитраектории по Важевскому эквивалентны, если отображение $F:[a, b] \times \mathbb{R}^{n} \rightarrow \operatorname{comp}\left[\mathbb{R}^{n}\right]$ непрерьвно по второму аргументу. В то же время для квазирешений можно доказать основное свойство квазитраекторий (см. [1], [2], [5]) при более общих предположениях, не предполагая непрерывность $F(\cdot, \cdot)$ по второму аргументу. Для этого достаточно предположить суперпозиционную измеримость отображения $F(\cdot, \cdot)$ (см. [6], [7]).

Скажем, что многозначное отображение $\widetilde{F}:[a, b] \times \mathbb{R}^{n} \times[0, \infty) \rightarrow \operatorname{comp}\left[\mathbb{R}^{n}\right]$ aппроксимирует отображение $F:[a, b] \times \mathbb{R}^{n} \rightarrow \operatorname{comp}\left[\mathbb{R}^{n}\right]$, если найдется такая функция $\xi(\cdot, \cdot, \cdot) \in K\left([a, b] \times \mathbb{R}^{n} \times[0, \infty)\right)$, что при почти всех $t \in[a, b]$ и всех $(x, \delta) \in \mathbb{R}^{n} \times[0, \infty)$ выполняется оценка

$$
h[F(t, x), \widetilde{F}(t, x, \delta)] \leqslant \xi(t, x, \delta) .
$$

Отображение $\widetilde{F}(\cdot, \cdot, \cdot)$ назовем аппроксимирующим отображсение $F(\cdot, \cdot)$ или просто аппроксимирующим. Функция $\xi(\cdot, \cdot, \cdot) \in K\left([a, b] \times \mathbb{R}^{n} \times[0, \infty)\right)$ в неравенстве (3) определяет степень близости значения $\widetilde{F}(t, x, \delta)$ в точке $(t, x) \in[a, b] \times$ $\mathbb{R}^{n}$ к значению $F(t, x)$ для каждого фиксированного $\delta \in[0, \infty)$. Эту функцию $\xi(\cdot, \cdot, \cdot)$ будем называть степенью аппроксимации отображсения $F:[a, b] \times$ $\mathbb{R}^{n} \rightarrow \operatorname{comp}\left[\mathbb{R}^{n}\right]$ отображсением $\widetilde{F}:[a, b] \times \mathbb{R}^{n} \times[0, \infty) \rightarrow \operatorname{comp}\left[\mathbb{R}^{n}\right]$ или просто степенью аппроксимации.

Отметим, что если для отображения $\widetilde{F}:[a, b] \times \mathbb{R}^{n} \times[0, \infty) \rightarrow \operatorname{comp}\left[\mathbb{R}^{n}\right]$ функция $\widetilde{\xi}:[a, b] \times \mathbb{R}^{n} \times[0, \infty) \rightarrow[0, \infty)$ определена равенством

$$
\widetilde{\xi}(t, x, \delta)=h[F(t, x), \widetilde{F}(t, x, \delta)]
$$

и эта функция принадлежит множеству $K\left([a, b] \times \mathbb{R}^{n} \times[0, \infty)\right)$, то отображение $\widetilde{F}:[a, b] \times \mathbb{R}^{n} \times[0, \infty) \rightarrow \operatorname{comp}\left[\mathbb{R}^{n}\right]$ аппроксимирует отображение $F:[a, b] \times$ $\mathbb{R}^{n} \rightarrow \operatorname{comp}\left[\mathbb{R}^{n}\right]$. При этом функция $\widetilde{\xi}(\cdot, \cdot, \cdot)$, определенная равенством $(4)$, является точной нижней гранью всех функций $\xi(\cdot, \cdot, \cdot) \in K\left([a, b] \times \mathbb{R}^{n} \times[0, \infty)\right)$, удовлетворяющих при почти всех $t \in[a, b]$ и всех $(x, \delta) \in \mathbb{R}^{n} \times[0, \infty)$ неравенству (3). 
Поэтому функцию $\widetilde{\xi}(\cdot, \cdot, \cdot) \in K\left([a, b] \times \mathbb{R}^{n} \times[0, \infty)\right)$ для отображения $\widetilde{F}(\cdot, \cdot, \cdot)$ можно считать "идеальной" степенью аппроксимации. Отметим, что иногда установить “идеальную" степень аппроксимации $\widetilde{\xi}(\cdot, \cdot, \cdot) \in K\left([a, b] \times \mathbb{R}^{n} \times[0, \infty)\right)$ для аппроксимирующего отображения $\widetilde{F}:[a, b] \times \mathbb{R}^{n} \times[0, \infty) \rightarrow \operatorname{comp}\left[\mathbb{R}^{n}\right]$ сложно. Гораздо проше найти "какую-нибудь" степень аппроксимации $\xi(\cdot, \cdot, \cdot) \in K([a, b] \times$ $\left.\mathbb{R}^{n} \times[0, \infty)\right)$, тем более, что во многих случаях этого достаточно, чтобы установить те или иные свойства множества решений дифференциального включения (см. ниже теоремы 1-6).

Отметим, что из определения аппроксимируюшего отображения $\widetilde{F}(\cdot, \cdot \cdot, \cdot)$ при почти всех $t \in[a, b]$ и всех $(x, \delta) \in \mathbb{R}^{n} \times[0, \infty)$ вытекает оценка

$$
\|\widetilde{F}(t, x, \delta)\| \leqslant\|F(t, x)\|+\xi(t, x, \delta) .
$$

Поэтому для каждого $U \in \operatorname{comp}\left[\mathbb{R}^{n}\right]$ и $\delta \in[0, \infty)$ для отображения $\widetilde{F}(\cdot, \cdot, \cdot)$ существует такая суммируемая функция $m_{U, \delta}:[a, b] \rightarrow[0, \infty)$, что при почти всех $t \in[a, b]$ и всех $x \in U$ и $\tau \in[0, \delta]$ имеет место неравенство $\|\widetilde{F}(t, x, \tau)\| \leqslant m_{U, \delta}(t)$.

Пару $(\widetilde{F}(\cdot, \cdot, \cdot), \xi(\cdot, \cdot, \cdot))$ будем назьвать аппроксимацией отображсения $F(\cdot, \cdot)$ или просто аппроксимацией. Если $\xi(\cdot, \cdot, \cdot)=0$, то в этом случае для любых $(t, x, \delta) \in[a, b] \times \mathbb{R}^{n} \times[0, \infty)$ справедливо равенство $\widetilde{F}(t, x, \delta)=F(t, x)$ и поэтому $(\widetilde{F}(\cdot, \cdot, \cdot), 0)$ будем называть “идеальной аппроксимацией”. Пару $(\widetilde{F}(\cdot, \cdot, \cdot), \xi(\cdot, \cdot, \cdot))$ будем назьвать аппроксим ацией вложсением, если при почти всех $t \in[a, b]$ и всех $(x, \delta) \in \mathbb{R}^{n} \times[0, \infty)$ выполняется включение $F(t, x) \subset$ $\widetilde{F}(t, x, \delta)$.

Для заданной степени аппроксимации $\xi(\cdot, \cdot, \cdot) \in K\left([a, b] \times \mathbb{R}^{n} \times[0, \infty)\right)$ отображение $\widetilde{F}:[a, b] \times \mathbb{R}^{n} \times[0, \infty) \rightarrow \operatorname{comp}\left[\mathbb{R}^{n}\right]$, аппроксимирующее отображение $F:[a, b] \times \mathbb{R}^{n} \rightarrow \operatorname{comp}\left[\mathbb{R}^{n}\right]$, можно построить, например, взяв для каждых $(t, x) \in[a, b] \times \mathbb{R}^{n}$ конечное множество всех центров, принадлежащих значению $F(t, x)$, таких, что шары с этими центрами, имеющие равные радиусы, покрывают множество $F(t, x)$. При этом значение $\xi(t, x, \delta)$ является радиусами этих шаров или оценкой этих радиусов. Данный пример показывает, что каждое значение многозначного отображения $\widetilde{F}:[a, b] \times \mathbb{R}^{n} \times[0, \infty) \rightarrow \operatorname{comp}\left[\mathbb{R}^{n}\right]$, аппроксимирующее отображение $F:[a, b] \times \mathbb{R}^{n} \rightarrow \operatorname{comp}\left[\mathbb{R}^{n}\right]$, при каждом $\delta \in[0, \infty)$ может иметь достаточно "простую структуру". Будем считать, что $\widetilde{F}(\cdot, \cdot \cdot, \cdot)$ определяет способ или метод аппроксимации отображения $F(\cdot, \cdot)$.

Отметим, что сами значения аппроксимирующего отображения $\widetilde{F}(\cdot, \cdot, \cdot)$ могут вычисляться с некоторой степенью точности (например, в рассмотренном выше примере центры шаров, покрывающие значения $\left.F(t, x),(t, x) \in[a, b] \times \mathbb{R}^{n}\right)$, которую можно задать некоторой функцией $\eta(\cdot, \cdot, \cdot) \in K\left([a, b] \times \mathbb{R}^{n} \times[0, \infty)\right)$. В связи с этим рассмотрим отображение $Q_{\eta}:[a, b] \times \mathbb{R}^{n} \times[0, \infty) \rightarrow \operatorname{comp}\left[\mathbb{R}^{n}\right]$, определенное равенством

$$
Q_{\eta}(t, x, \delta)=\widetilde{F}(t, x, \delta)^{\eta(t, x, \delta)},
$$

где функция $\eta(\cdot, \cdot, \cdot) \in K\left([a, b] \times \mathbb{R}^{n} \times[0, \infty)\right)$ в каждой точке $(t, x) \in[a, b] \times \mathbb{R}^{n}$ при каждом фиксированном $\delta \in[0, \infty)$ определяет погрешность вычисления значений аппроксимируюшего отображения $\widetilde{F}(\cdot, \cdot, \cdot)$, причем эти погрешности могут быть неравномерны относительно фазовой переменной $x \in \mathbb{R}^{n}$. Далее, функцию $\eta(\cdot, \cdot, \cdot)$ будем назьвать радиусом внешних возмущений аппроксимирующего отображсения $\widetilde{F}(\cdot, \cdot, \cdot)$ или просто радиусом внешних возмушений. 
Заметим, что значения отображения $Q_{\eta}(\cdot, \cdot, \cdot)$, заданного равенством $(6)$, могут быть невыпукльми множествами даже в том случае, когда значения отображения $F(\cdot, \cdot)$ - выпуклые множества. Кроме того, из определения отображения $Q_{\eta}(\cdot, \cdot, \cdot)$ и неравенства $(3)$ при почти всех $t \in[a, b]$ и всех $(x, \delta) \in \mathbb{R}^{n} \times[0, \infty)$ следует оценка

$$
h\left[F(t, x), Q_{\eta}(t, x, \delta)\right] \leqslant \xi(t, x, \delta)+\eta(t, x, \delta) .
$$

Таким образом, из оценки (7) вытекает, что для каждой функции $\eta(\cdot, \cdot, \cdot) \in$ $K\left([a, b] \times \mathbb{R}^{n} \times[0, \infty)\right)$ при почти всех $t \in[a, b]$ и всех $x \in \mathbb{R}^{n}$ справедливо равенство

$$
\lim _{\delta \rightarrow 0+0} h\left[F(t, x), Q_{\eta}(t, x, \delta)\right]=0 .
$$

Поэтому для заданной аппроксимации $(\widetilde{F}(\cdot, \cdot, \cdot), \xi(\cdot, \cdot, \cdot))$ все отображения $Q_{\eta}:[a, b] \times \mathbb{R}^{n} \times[0, \infty) \rightarrow \operatorname{comp}\left[\mathbb{R}^{n}\right]$, определенные равенством (6) и зависящие от функции $\eta(\cdot, \cdot, \cdot) \in K\left([a, b] \times \mathbb{R}^{n} \times[0, \infty)\right)$, близки (в смысле равенства $\left.(8)\right)$ к отображению $F:[a, b] \times \mathbb{R}^{n} \rightarrow \operatorname{comp}\left[\mathbb{R}^{n}\right]$, определяющему дифференциальное включение (1).

Пусть $\eta(\cdot, \cdot, \cdot) \in K\left([a, b] \times \mathbb{R}^{n} \times[0, \infty)\right)$. Рассмотрим при каждом фиксированном $\delta \in[0, \infty)$ дифференциальное включение

$$
\dot{x}(t) \in Q_{\eta}(t, x(t), \delta), \quad t \in[a, b]
$$

где отображение $Q_{\eta}:[a, b] \times \mathbb{R}^{n} \times[0, \infty) \rightarrow \operatorname{comp}\left[\mathbb{R}^{n}\right]$ задано равенством (6). Отметим, что правая часть дифференциального включения (9) может быть и невыпуклозначной. Дифференциальное включение (9) будем называть аппроксимирующим дифференциальное включение (1) с внешними возмущениями.

Каждое решение $x:[a, b] \rightarrow \mathbb{R}^{n}$ дифференциального включения (9) будем называть $\delta$-решением (приближенньм решением с точностью до $\delta$ или просто приближенным решением) включения (1).

Рассмотрим также дифференциальное включение

$$
\dot{x}(t) \in \operatorname{co} F(t, x(t)), \quad t \in[a, b] .
$$

Пусть $V \subset C^{n}[a, b]$. Обозначим через $H(V) \quad\left(H_{\eta(\delta)}(V)\right)$ множество решений $\left(\delta\right.$-решений) включения (1), а через $H_{\text {со }}(V)$ - множество решений включения $(10)$, принадлежащие заданному множеству $V$.

Будем говорить, что для дифференциального включения (1) на множестве $V \subset$ $C^{n}[a, b]$ выполняется принцип плотности, если имеет место равенство

$$
\overline{H(V)}=H_{\mathrm{co}}(V)
$$

где $\overline{H(V)}$ - замыкание в пространстве $C^{n}[a, b]$ множества $H(V)$. 
ЗАмЕЧАНИЕ 2. Отметим, что принцип плотности выполняется не всегда. Это показывает пример Плиса (A.Plis) (см. [8], [1]). Первые достаточные условия, когда выполняется равенство (11), для задачи Коши были получены А.Ф. Филипповьгм (см. [9], [10]), а для периодических решений и для краевых задач эти условия получены в работах [7]; [11]; [12]. Принцип плотности, как отмечено Пианижиани (G.Pianigiani), является фундаментальным свойством для дифференциальных включений с невыпуклой правой частью (см. [13]). Здесь доказано, что и в аппроксимации дифференциальных включений с невыпуклой правой частью он играет важную роль.

Пусть $\psi(\cdot, \cdot, \cdot) \in \widetilde{K}\left([a, b] \times \mathbb{R}^{n} \times[0, \infty)\right)$. Определим функцию $\varphi(\psi):[a, b] \times$ $R^{n} \times[0, \infty) \rightarrow[0, \infty)$ равенством

$$
\varphi(\psi)(t, x, \delta)=\sup _{y \in B[x, \psi(t, x, \delta)]} h[F(t, x), F(t, y)]
$$

Значения функции $\varphi(\psi)(\cdot, \cdot, \cdot)($ см. [14]) в точке $(t, x, \delta)$ будем называть модулем непрерывности отображения $F:[a, b] \times \mathbb{R}^{n} \rightarrow \operatorname{comp}\left[\mathbb{R}^{n}\right]$ в точке $(t, x)$ по переменной $x$ в шаре $B[x, \psi(t, x, \delta)]$, функцию $\psi(\cdot, \cdot, \cdot)$ - функцией радиуса модуля непрерывности или просто радиусом непрерывности, а саму функцию $\varphi(\psi)(\cdot, \cdot, \cdot)$ - функиией модуля непрерывности или просто модулем непрерывности отображения $F:[a, b] \times \mathbb{R}^{n} \rightarrow \operatorname{comp}\left[\mathbb{R}^{n}\right]$ относительно радиуса непрерывности $\psi(\cdot, \cdot, \cdot)$. Далее, для сокращения записи будем коротко писать $\varphi(\psi)(\cdot, \cdot, \cdot \cdot)$ - модуль непрерывности отображения $F(\cdot, \cdot)$.

Аналогично лемме 3 из [14] доказывается

Лемма 1. Пусть $\psi(\cdot, \cdot, \cdot) \in \widetilde{K}\left([a, b] \times \mathbb{R}^{n} \times[0, \infty)\right)$.

Тогда функиия $\varphi(\psi)(\cdot, \cdot, \cdot)$, определенная равенством (12), принадлежит мнохеству $K\left([a, b] \times \mathbb{R}^{n} \times[0, \infty)\right)$.

Пусть $V \subset C^{n}[a, b]$. Обозначим

$$
U(V)=\left\{x \in \mathbb{R}^{n}: \exists y(\cdot) \in V, \quad t \in[a, b] \quad x=y(t)\right\} .
$$

Теорема 1. Пусть $V \subset C^{n}[a, b]$ - ограниченное замкнутое множество пространства $C^{n}[a, b]$, и пусть $\psi(\cdot, \cdot, \cdot) \in P\left([a, b] \times \mathbb{R}^{n} \times[0, \infty)\right)$. Далее, пусть пара $(\widetilde{F}(\cdot, \cdot, \cdot), \xi(\cdot, \cdot, \cdot))$ аппроксимирует отображсение $F(\cdot, \cdot)$. Тогда для любой функиии $\eta(\cdot, \cdot, \cdot) \in K\left([a, b] \times \mathbb{R}^{n} \times[0, \infty)\right)$, для которой существует такое число $\varepsilon>0$, что при почти всех $t \in[a, b]$, всех $x \in(U(V))^{\varepsilon}$ (cм. (13)) и $\delta \in[0, \infty)$ имеет место неравенство

$$
\xi(t, x, \delta)+\varphi(\psi)(t, x, \delta) \leqslant \eta(t, x, \delta),
$$

где $\varphi(\psi)(\cdot, \cdot, \cdot)$-модуль непрерьвности, а $\xi(\cdot, \cdot, \cdot)$ - степень аппроксимации отображения $F(\cdot, \cdot)$, выполняется соотношение

$$
H_{\mathrm{co}}(V)=\bigcap_{\delta>0} \overline{H_{\eta(\delta)}\left(V^{\delta}\right)},
$$

дде $\overline{H_{\eta(\delta)}\left(V^{\delta}\right)}$ - замыкание в пространстве $C^{n}[a, b]$ множсества $H_{\eta(\delta)}\left(V^{\delta}\right)$, $V^{\delta}$ - замкнутая в пространстве $C^{n}[a, b]$-окрестность мнохсества $V$. 
ДокаЗАТЕЛЬСтво. Пусть $\varepsilon>0$. И пусть функция $\eta(\cdot, \cdot, \cdot) \in K\left([a, b] \times \mathbb{R}^{n} \times\right.$ $[0, \infty))$ при почти всех $t \in[a, b]$, всех $x \in(U(V))^{\varepsilon}$ и $\delta \in[0, \infty)$ удовлетворяет неравенству (14). Вначале докажем, что

$$
H_{\mathrm{co}}(V) \subset \bigcap_{\delta>0} \overline{H_{\eta(\delta)}\left(V^{\delta}\right)}
$$

Пусть $x(\cdot) \in H_{\text {со }}(V)$ и $\delta>0$. Согласно [6], [7] для $x(\cdot)$ найдется такая последовательность абсолютно непрерывных функций $x_{i}:[a, b] \rightarrow \mathbb{R}^{n}, i=1,2, \ldots$, обладаюших свойствами: $x_{i}(\cdot) \rightarrow x(\cdot)$ в $C^{n}[a, b]$ при $i \rightarrow \infty$ и для любого $i=1,2, \ldots$ $\dot{x}_{i}(\cdot) \in S(F(\cdot, x(\cdot)))$. Пусть $I_{1}$ - такой номер, что при всех $i \geqslant I_{1}$ выполняется включение $x_{i}(\cdot) \in V^{\delta}$ и при всех $i \geqslant I_{1}$ и $t \in[a, b]$ справедливо соотношение $x_{i}(t) \in(U(V))^{\varepsilon}$. Так как $\psi(\cdot, \cdot, \cdot) \in P\left([a, b] \times \mathbb{R}^{n} \times[0, \infty)\right)$, то найдется такой номер $I_{2}$, что при всех $i \geqslant I_{2}$ при почти всех $t \in[a, b]$ имеет место включение $x(t) \in B\left[x_{i}(t), \psi\left(t, x_{i}(t), \delta\right)\right]$. Тогда при всех $i \geqslant \max \left\{I_{1}, I_{2}\right\}$ при почти всех $t \in[a, b]$ справедливы оценки

$$
\begin{aligned}
& \rho\left[\dot{x}_{i}(t), \widetilde{F}\left(t, x_{i}(t), \delta\right)\right] \leqslant h\left[F(t, x(t)), \widetilde{F}\left(t, x_{i}(t), \delta\right)\right] \\
& \quad \leqslant h\left[F\left(t, x_{i}(t)\right), F(t, x(t))\right]+h\left[F\left(t, x_{i}(t)\right), \widetilde{F}\left(t, x_{i}(t), \delta\right)\right] \\
& \quad \leqslant \varphi(\psi)\left(t, x_{i}(t), \delta\right)+\xi\left(t, x_{i}(t), \delta\right) \leqslant \eta\left(t, x_{i}(t), \delta\right) .
\end{aligned}
$$

Таким образом, для любого $i \geqslant \max \left\{I_{1}, I_{2}\right\}$ имеет место включение $x_{i}(\cdot) \in$ $H_{\eta(\delta)}\left(V^{\delta}\right)$. Это означает, что $x(\cdot)$ - предельная точка множества $H_{\eta(\delta)}\left(V^{\delta}\right)$ и поэтому $x(\cdot) \in \overline{H_{\eta(\delta)}\left(V^{\delta}\right)}$. Следовательно, $x(\cdot) \in \bigcap_{\delta>0} \overline{H_{\eta(\delta)}\left(V^{\delta}\right)}$ и вложение (16) доказано.

Теперь докажем, что

$$
\bigcap_{\delta>0} \overline{H_{\eta(\delta)}\left(V^{\delta}\right)} \subset H_{\mathrm{co}}(V) .
$$

Пусть $x(\cdot) \in \bigcap_{\delta>0} \overline{H_{\eta(\delta)}\left(V^{\delta}\right)}$, и пусть $\delta_{i} \rightarrow 0$, где $\delta_{i}$ - последовательность положительных чисел. Далее, пусть $x_{i}(\cdot) \in H_{\eta\left(\delta_{i}\right)}\left(V^{\delta_{i}}\right), i=1,2, \ldots,-$ такая последовательность, что $x_{i}(\cdot) \rightarrow x(\cdot)$ в $C^{n}[a, b]$ при $i \rightarrow \infty$. Так как последовательность $\dot{x}_{i}(\cdot), i=1,2, \ldots$, ограничена суммируемой функцией, то $x(\cdot)$ абсолютно непрерывна и $\dot{x}_{i}(\cdot) \rightarrow \dot{x}(\cdot)$ слабо в $L^{n}[a, b]$ при $i \rightarrow \infty$.

Определим последовательность абсолютно непрерывных функций $y_{i}:[a, b] \rightarrow$ $\mathbb{R}^{n}, i=1,2, \ldots$, для каждого $i=1,2, \ldots$ удовлетворяюшую включению $\dot{y}_{i}(\cdot) \in$ $S(F(\cdot, x(\cdot)))$, равенству $y_{i}(a)=x_{i}(a)$ и при почти всех $t \in[a, b]$ соотношению

$$
\left|\dot{x}_{i}(t)-\dot{y}_{i}(t)\right|=\rho\left[\dot{x}_{i}(t), F(t, x(t))\right] .
$$

Тогда для любого $i=1,2, \ldots$ при почти всех $t \in[a, b]$ получаем оценки

$$
\begin{aligned}
& \left|\dot{x}_{i}(t)-\dot{y}_{i}(t)\right| \leqslant h\left[Q_{\eta}\left(t, x_{i}(t), \delta_{i}\right), \widetilde{F}\left(t, x_{i}(t), \delta_{i}\right)\right] \\
& \quad+h\left[\widetilde{F}\left(t, x_{i}(t), \delta_{i}\right), F\left(t, x_{i}(t)\right)\right]+h\left[F\left(t, x_{i}(t)\right), F(t, x(t))\right] .
\end{aligned}
$$

Таким образом, для любого $i=1,2, \ldots$ при почти всех $t \in[a, b]$ имеет место неравенство

$$
\left|\dot{x}_{i}(t)-\dot{y}_{i}(t)\right| \leqslant \eta\left(t, x_{i}(t), \delta_{i}\right)+\xi\left(t, x_{i}(t), \delta_{i}\right)+h\left[F\left(t, x_{i}(t)\right), F(t, x(t))\right] .
$$


Из неравенства (18) и теоремы Лебега следует, что

$$
\lim _{i \rightarrow \infty}\left\|\dot{x}_{i}-\dot{y}_{i}\right\|_{L}=0
$$

Из этого вытекает, что последовательность $y_{i}(\cdot) \rightarrow x(\cdot)$ в $C^{n}[a, b]$ при $i \rightarrow \infty$ и $\dot{y}_{i}(\cdot) \rightarrow \dot{x}(\cdot)$ слабо в пространстве $L^{n}[a, b]$ при $i \rightarrow \infty$. Так как для любого $i=1,2, \ldots \dot{y}_{i}(\cdot) \in S(F(\cdot, x(\cdot)))$, то согласно [4] $\dot{x}(\cdot) \in S(\operatorname{co} F(\cdot, x(\cdot)))$, т.е. $x(\cdot) \in H_{\mathrm{co}}(V)$. Таким образом, вложение (17) доказано. И, следовательно, равенство (15) справедливо. Теорема доказана.

ЗАмЕчАНИЕ 3. Из теоремы 1 и леммы 1 вытекает, что для любой степени аппроксимации $\xi(\cdot, \cdot, \cdot)$ и заданного модуля непрерьвности $\varphi(\psi)(\cdot, \cdot \cdot, \cdot)$ отображения $F(\cdot, \cdot)$, найдется такой радиус внешних возмушений $\eta(\cdot, \cdot, \cdot) \in K([a, b] \times$ $\left.\mathbb{R}^{n} \times[0, \infty)\right)$ аппроксимируюшего отображения $\widetilde{F}(\cdot, \cdot, \cdot)$, для которого выполняется равенство (15). Для этого достаточно положить $\eta(\cdot, \cdot, \cdot)=\xi(\cdot, \cdot, \cdot)+$ $\varphi(\psi)(\cdot, \cdot, \cdot)$. Отметим также, что модуль непрерьвности $\varphi(\psi)(\cdot, \cdot, \cdot)$ отображения $F(\cdot, \cdot)$ зависит от радиуса непрерьвности $\psi(\cdot, \cdot, \cdot)$ и поэтому, подбирая радиус $\psi(\cdot, \cdot, \cdot)$, можно величину $\varphi(\psi)(\cdot, \cdot, \cdot)$ сделать достаточно малой. Таким образом, другими словами, теорема 1 утверждает, что если внешние возмущения $\eta(\cdot, \cdot, \cdot) \in K\left([a, b] \times \mathbb{R}^{n} \times[0, \infty)\right)$ сравнимы со степенью аппроксимации $\xi(\cdot, \cdot, \cdot) \in K\left([a, b] \times \mathbb{R}^{n} \times[0, \infty)\right)$ отображения $F(\cdot, \cdot)$, то справедливо равенство (15).

Теорема 2. Пусть $V$ - ограниченное замкнутое множество пространства $C^{n}[a, b]$. Пусть пара $(\widetilde{F}(\cdot, \cdot, \cdot), \xi(\cdot, \cdot, \cdot))$ аппроксимирует отображение $F(\cdot, \cdot)$. Тогда для того чтобъ для любой функиии $\eta(\cdot, \cdot, \cdot) \in K([a, b] \times$ $\left.\mathbb{R}^{n} \times[0, \infty)\right)$ при почти всех $t \in[a, b]$ и всех $(x, \delta) \in \overline{U(V)} \times[0, \infty)($ см. (13)) удовлетворяющей соотночению $\xi(t, x, \delta) \leqslant \eta(t, x, \delta)$ имело место равенство

$$
\overline{H(V)}=\bigcap_{\delta>0} \overline{H_{\eta(\delta)}\left(V^{\delta}\right)}
$$

әде $\overline{H(V)}$ - замыкание мнохества $H(V)$ в пространстве $C^{n}[a, b]$, необходимо и достаточно, чтобь для включения (1) на множестве $V$ выполнялся приниип плотности.

ДокАЗАТЕЛЬство. Необходимость непосредственно вытекает из теоремы 1 (см. замечание 3 ).

Достаточность. Пусть имеет место равенство (11). Покажем вначале, что

$$
\overline{H(V)} \subset \bigcap_{\delta>0} \overline{H_{\eta(\delta)}\left(V^{\delta}\right)}
$$

Пусть $x(\cdot) \in \overline{H(V)}$ и $\delta>0$. Пусть последовательность функций $x_{i}(\cdot) \in H(V)$ обладает свойством: $x_{i}(\cdot) \rightarrow x(\cdot)$ в $C^{n}[a, b]$ при $i \rightarrow \infty$. Тогда для любого $i=1,2, \ldots$ при почти всех $t \in[a, b]$ имеют место неравенства

$$
\rho\left[\dot{x}_{i}(t), \widetilde{F}\left(t, x_{i}(t), \delta\right)\right] \leqslant h\left[F\left(t, x_{i}(t)\right), \widetilde{F}\left(t, x_{i}(t), \delta\right)\right] \leqslant \xi\left(t, x_{i}(t), \delta\right) \leqslant \eta\left(t, x_{i}(t), \delta\right) .
$$


Таким образом, при любом $i=1,2, \ldots x_{i}(\cdot) \in H_{\eta(\delta)}\left(V^{\delta}\right)$ и, значит, $x(\cdot) \in$ $\overline{H_{\eta(\delta)}\left(V^{\delta}\right)}$. Поэтому $x(\cdot) \in \bigcap_{\delta>0} \overline{H_{\eta(\delta)}\left(V^{\delta}\right)}$. Следовательно, вложение (20) справедливо.

Включение

$$
\bigcap_{\delta>0} \overline{H_{\eta(\delta)}\left(V^{\delta}\right)} \subset \overline{H(V)}
$$

в силу равенства (11) доказывается точно так же, как и в теореме 1. Таким образом, равенство (19) имеет место. Теорема доказана.

ЗАмечАниЕ 4. Отметим, что теорема 2 вместе с примером Плиса (см. [8]; [1]) доказывает, что если внешние возмушения $\eta(\cdot, \cdot, \cdot) \in K\left([a, b] \times \mathbb{R}^{n} \times[0, \infty)\right)$ больше или равны степени аппроксимации $\xi(\cdot, \cdot, \cdot) \in K\left([a, b] \times \mathbb{R}^{n} \times[0, \infty)\right)$ отображения $F:[a, b] \times \mathbb{R}^{n} \rightarrow \operatorname{comp}\left[\mathbb{R}^{n}\right]$, не обладающего свойством вьпуклости значений, то аппроксимация дифференциального включения (1), вообще говоря, может и не быть устойчивой, т.е. "небольшие" изменения правой части могут существенно изменить множество решений.

Будем говорить, что отображение $F:[a, b] \times \mathbb{R}^{n} \rightarrow \operatorname{comp}\left[\mathbb{R}^{n}\right]$ удовлетворяет условию Липшица, если сушествует такая суммируемая функция $l:[a, b] \rightarrow[0, \infty)$, что при почти всех $t \in[a, b]$ и всех $x, y \in \mathbb{R}^{n}$ справедливо равенство

$$
h[F(t, x), F(t, y)] \leqslant l(t)|x-y|
$$

Будем говорить, что функция $q:[a, b] \times[0, \infty) \rightarrow[0, \infty)$ обладает свойством $\mathrm{A}$, если для любого $\delta \in[0, \infty)$ функция $q(\cdot, \delta)$ измерима и для любого $\delta \in[0, \infty)$ найдется такая суммируемая функция $m_{\delta}:[a, b] \rightarrow[0, \infty)$, что при почти всех $t \in$ $[a, b]$ и всех $\tau \in[0, \delta]$ выполняется неравенство $q(t, \tau) \leqslant m_{\delta}(t)$.

Будем говорить, что отображение $\widetilde{F}:[a, b] \times \mathbb{R}^{n} \times[0, \infty) \rightarrow \operatorname{comp}\left[\mathbb{R}^{n}\right]$ обладает свойством $\mathrm{B}$, если при всех $(x, \delta) \in \mathbb{R}^{n} \times[0, \infty)$ отображение $\widetilde{F}(\cdot, x, \delta)$ измеримо и сушествует такая функция $q:[a, b] \times[0, \infty) \rightarrow[0, \infty)$, обладаюшая свойством А, что при почти всех $t \in[a, b]$ и всех $x, y \in \mathbb{R}^{n}$ и $\delta \in[0, \infty)$ вьполняется неравенство

$$
h[\widetilde{F}(t, x, \delta), \widetilde{F}(t, y, \delta)] \leqslant q(t, \delta)|x-y| .
$$

Пусть $M \in \operatorname{comp}\left[\mathbb{R}^{n}\right]$, и пусть $\eta(\cdot, \cdot, \cdot) \in K\left([a, b] \times \mathbb{R}^{n} \times[0, \infty)\right)$. Обозначим через $H(M)$ и $H_{\eta(\delta)}(M)$ множество решений задачи Коши включений (1) и $(9)$, начальные значения которых принадлежат множеству $M$ соответственно.

Tеорема 3. Пусть $M \in \operatorname{comp}\left[\mathbb{R}^{n}\right]$, u nyсmь napa $(\widetilde{F}(\cdot, \cdot, \cdot), \xi(\cdot, \cdot, \cdot))$ annpoксимирует отображсение $F(\cdot, \cdot)$. Далее, пусть отображсение $F(\cdot, \cdot)$ удовлетворяет условию Липиица, а отображсние $\widetilde{F}(\cdot, \cdot, \cdot)$ обладает свойством В. Тогда для любой функиии $\eta(\cdot, \cdot, \cdot) \in K\left([a, b] \times \mathbb{R}^{n} \times[0, \infty)\right)$ имеют место равенства

$$
\overline{H(M)}=\bigcap_{\delta>0} \overline{H_{\eta(\delta)}(M)}=\bigcap_{\delta>0} \overline{H_{\eta(\delta)}\left(M^{\delta}\right)},
$$

әде $\overline{H(M)}, \overline{H_{\eta(\delta)}(M)}, \overline{H_{\eta(\delta)}\left(M^{\delta}\right)}$ - замыкания в пространстве $C^{n}[a, b]$ coответствующих мнохеств. 
ДокАЗАТЕльство. Вначале покажем вложение

$$
\bigcap_{\delta>0} \overline{H_{\eta(\delta)}(M)} \subset \overline{H(M)}
$$

Действительно, пусть $x(\cdot) \in \bigcap_{\delta>0} \overline{H_{\eta(\delta)}(M)}$, и пусть последовательности $x_{i}(\cdot) \in$ $H_{\eta\left(\delta_{i}\right)}(M)$ и $\delta_{i}>0, i=1,2, \ldots$, обладают свойством: $x_{i}(\cdot) \rightarrow x(\cdot)$ в $C^{n}[a, b]$ и $\delta_{i} \rightarrow 0$ при $i \rightarrow \infty$. Тогда в силу неравенства (7) для каждого $i=1,2, \ldots$ при почти всех $t \in[a, b]$ выполняется соотношение

$$
\rho\left[\dot{x}_{i}(t), F\left(t, x_{i}(t)\right)\right] \leqslant \xi\left(t, x_{i}(t), \delta_{i}\right) .
$$

Поэтому согласно [9], [10] для каждого $i=1,2, \ldots$ найдется $y_{i}(\cdot) \in H(M)$, для которого при всех $t \in[a, b]$ имеет место оценка

$$
\left|x_{i}(t)-y_{i}(t)\right| \leqslant \int_{a}^{t}\left(\exp \int_{s}^{t} l(\tau) d \tau\right) \xi\left(s, x_{i}(s), \delta_{i}\right) d s
$$

где функция $l(\cdot)$ удовлетворяет неравенству $(21)$. Так как $\xi(\cdot, \cdot, \cdot) \in K([a, b] \times$ $\mathbb{R}^{n} \times[0, \infty)$, то из оценки $(25)$ следует, что $y_{i}(\cdot) \rightarrow x(\cdot)$ в $C^{n}[a, b]$ при $i \rightarrow \infty$. Поэтому $x(\cdot) \in \overline{H(M)}$ и, следовательно, вложение (24) доказано.

Аналогично доказывается включение

$$
\overline{H(M)} \subset \bigcap_{\delta>0} \overline{H_{\eta(\delta)}(M)}
$$

Таким образом, равенство

$$
\overline{H(M)}=\bigcap_{\delta>0} \overline{H_{\eta(\delta)}(M)}
$$

справедливо. Второе равенство в (23) доказывается точно так же. Теорема доказана.

Если пара $(\widetilde{F}(\cdot, \cdot, \cdot), \xi(\cdot, \cdot, \cdot))$ аппроксимирует отображение $F:[a, b] \times \mathbb{R}^{n} \rightarrow$ $\operatorname{comp}\left[\mathbb{R}^{n}\right]$ вложением, теоремы 1,2 можно уточнить.

ТЕОРема 4. Пусть $V$ - ограниченное замкнутое множество пространства $C^{n}[a, b]$, и пусть $\psi(\cdot, \cdot, \cdot) \in P\left([a, b] \times \mathbb{R}^{n} \times[0, \infty)\right)$. Далее, пусть пара $(\widetilde{F}(\cdot, \cdot, \cdot), \xi(\cdot, \cdot, \cdot))$ аппроксимирует отображение $F(\cdot, \cdot)$ вложением. Тогда для любой функиии $\eta(\cdot, \cdot, \cdot) \in K\left([a, b] \times \mathbb{R}^{n} \times[0, \infty)\right)$, для которой существует такое число $\varepsilon>0$, что при почти всех $t \in[a, b]$, всех $x \in \overline{U(V)} \varepsilon$ (см. (13)) u $\delta \in[0, \infty)$ выполняется оценка

$$
\varphi(\psi)(t, x, \delta) \leqslant \eta(t, x, \delta),
$$

справедливо равенство (15). 
ДоказАтельство. Докажем вложение (16). Пусть $x(\cdot) \in H_{\text {со }}(V)$ и $\delta>0$. Тогда согласно [6], [7] для $x(\cdot)$ найдется такая последовательность абсолютно непрерывных функций $x_{i}:[a, b] \rightarrow \mathbb{R}^{n}, i=1,2, \ldots$, обладающая свойством: $x_{i}(\cdot) \rightarrow$ $x(\cdot)$ в $C^{n}[a, b]$ при $i \rightarrow \infty$ и для любого $i=1,2, \ldots \dot{x}_{i}(\cdot) \in S(F(\cdot, x(\cdot)))$. Пусть $I$ - такой номер, что для любого $i \geqslant I$ выполняются включения $x_{i}(\cdot) \in V^{\varepsilon}, x_{i} \in V^{\delta}$ и при почти всех $t \in[a, b]$ имеет место соотношение $x(t) \in B\left[x_{i}(t), \psi\left(t, x_{i}(t), \delta\right)\right]$. Тогда при всех $i \geqslant I$ и при почти всех $t \in[a, b]$ справедливы оценки

$$
\begin{aligned}
& \rho\left[\dot{x}_{i}(t), \widetilde{F}\left(t, x_{i}(t), \delta\right)\right] \leqslant h^{+}\left[F(t, x(t)) ; \widetilde{F}\left(t, x_{i}(t), \delta\right)\right] \\
& \quad \leqslant h^{+}\left[F(t, x(t)) ; F\left(t, x_{i}(t)\right)\right]+h^{+}\left[F\left(t, x_{i}(t)\right) ; \widetilde{F}\left(t, x_{i}(t), \delta\right)\right] \\
& \quad \leqslant h\left[F(t, x(t)), F\left(t, x_{i}(t)\right)\right] \leqslant \varphi(\psi)\left(t, x_{i}(t), \delta\right) \leqslant \eta\left(t, x_{i}(t), \delta\right) .
\end{aligned}
$$

Таким образом, для любого $i \geqslant I$ выполняется включение $x_{i}(\cdot) \in H_{\eta(\delta)}\left(V^{\delta}\right)$. Поэтому для любого $\delta>0$ имеет место включение $x(\cdot) \in \overline{H_{\eta(\delta)}\left(V^{\delta}\right)}$, т.е. $x(\cdot) \in$ $\bigcap_{\delta>0} \overline{H_{\eta(\delta)}\left(V^{\delta}\right)}$. Следовательно, вложение (16) доказано. Соотношение (17) доказывается аналогично теореме 1 . Теорема доказана.

Аналогично теореме 2 доказывается

ТЕОРема 5. Пусть $V$ - ограниченное замкнутое множество пространства $C^{n}[a, b]$. Далее, пусть пара $(\widetilde{F}(\cdot, \cdot, \cdot), \xi(\cdot, \cdot, \cdot))$ aпnроксимирует отображсние $F(\cdot, \cdot)$ вложением. Тогда для того чтобъ для любой функиии $\eta(\cdot, \cdot, \cdot) \in K\left([a, b] \times \mathbb{R}^{n} \times[0, \infty)\right)$ имело место равенство (19), необходимо и достаточно, чтобь для включения (1) на множестве $V$ выполнялся приниип плотности.

\section{§ 2. Аппроксимация с внутренними и внешними возмущениями}

2.1. Общий случай. Пусть $\eta_{0}(\cdot, \cdot, \cdot) \in \widetilde{K}\left([a, b] \times \mathbb{R}^{n} \times[0, \infty)\right)$. Определим функцию $\xi\left(\eta_{0}\right):[a, b] \times \mathbb{R}^{n} \times[0, \infty) \rightarrow[0, \infty)$ равенством

$$
\xi\left(\eta_{0}\right)(t, x, \delta)=\sup _{z \in B\left[x, \eta_{0}(t, x, \delta)\right]} \xi(t, z, \delta) .
$$

Лемма 2. Пусть $\eta_{0}(\cdot, \cdot, \cdot) \in \widetilde{K}\left([a, b] \times \mathbb{R}^{n} \times[0, \infty)\right)$. Тогда функиия $\xi\left(\eta_{0}\right)(\cdot, \cdot, \cdot)$, заданная равенством $(26)$, принадлежст множеству $K([a, b] \times$ $\left.\mathbb{R}^{n} \times[0, \infty)\right)$.

ДоказАТЕльство. Так как функция $\xi(\cdot, \cdot, \cdot) \in K\left([a, b] \times \mathbb{R}^{n} \times[0, \infty)\right)$, то при каждом $x \in \mathbb{R}^{n}$ и $\delta \in[0, \infty)$ функция $\xi\left(\eta_{0}\right)(\cdot, x, \delta)$ измерима и для каждого $U \in \operatorname{comp}\left[\mathbb{R}^{n}\right]$ и $\delta \in[0, \infty)$ найдется такая функция $m_{U, \delta}(\cdot) \in L^{1}[a, b]$, что при почти всех $t \in[a, b]$ выполняется неравенство $\xi\left(\eta_{0}\right)(t, x, \tau) \leqslant m_{U, \delta}(t)$.

Далее, будем рассматривать только такие точки $t \in[a, b]$, в которых функция $\xi(t, \cdot, \cdot)$ непрерывна по второму аргументу. Ниже покажем, что в каждой точке $t \in[a, b]$, в которой функции $\xi(t, \cdot, \cdot)$ и $\eta_{0}(t, \cdot, \cdot)$ непрерывны в точке $(x, \delta) \in$ $\mathbb{R}^{n} \times[0, \infty)$, непрерывна и функция $\xi\left(\eta_{0}\right)(t, \cdot, \cdot)$ в этой точке. Пусть точка $t \in$ $[a, b]$ обладает этим свойством. Вначале убедимся, что $\xi\left(\eta_{0}\right)(t, \cdot, \cdot)$ полунепрерывна сверху в точке $(x, \delta)$. Действительно, пусть последовательности $x_{i} \in \mathbb{R}^{n}$, 
$\delta_{i} \in[0, \infty), i=1,2, \ldots$, сходятся к $x$ и $\delta$ соответственно. Выбирая подпоследовательность, не уменьшая общности, можно считать, что

$$
\lim _{i \rightarrow \infty} \xi\left(\eta_{0}\right)\left(t, x_{i}, \delta_{i}\right)=\varlimsup_{i \rightarrow \infty} \xi\left(\eta_{0}\right)\left(t, x_{i}, \delta_{i}\right) .
$$

Пусть $y_{i} \in B\left[x_{i}, \eta_{0}\left(t, x_{i}, \delta_{i}\right)\right], i=1,2, \ldots,-$ такой элемент, для которого выполняется равенство

$$
\xi\left(\eta_{0}\right)\left(t, x_{i}, \delta_{i}\right)=\xi\left(t, y_{i}, \delta_{i}\right)
$$

Так как $\eta_{0}(\cdot, \cdot, \cdot) \in \widetilde{K}\left([a, b] \times \mathbb{R}^{n} \times[0, \infty)\right)$, то, не уменьшая общности, можно считать, что $y_{i} \rightarrow y$ в $\mathbb{R}^{n}$ при $i \rightarrow \infty$. Очевидно, что $y \in B\left[x, \eta_{0}(t, x, \delta)\right]$. Поэтому из равенств $(27)$ и $(28)$ вытекают соотношения

$$
\varlimsup_{i \rightarrow \infty} \xi\left(\eta_{0}\right)\left(t, x_{i}, \delta_{i}\right)=\xi(t, y, \delta) \leqslant \xi\left(\eta_{0}\right)(t, x, \delta) .
$$

Таким образом, отображение $\xi\left(\eta_{0}\right)(t, \cdot, \cdot)$ полунепрерывно сверху в точке $(x, \delta)$.

Аналогично доказывается, что $\xi\left(\eta_{0}\right)(t, \cdot, \cdot)$ полунепрерьвно снизу в $(x, \delta)$. Следовательно, $\xi\left(\eta_{0}\right)(t, \cdot, \cdot)$ непрерывно в точке $(x, \delta)$. Так как для любого $x \in \mathbb{R}^{n}$ справедливо равенство $\xi\left(\eta_{0}\right)(t, x, 0)=0$, то из доказанного следует, что при почти всех $t \in[a, b]$ и всех $x \in \mathbb{R}^{n}$ имеет место соотношение $\lim _{\delta \rightarrow 0+0}^{z \rightarrow x} \xi\left(\eta_{0}\right)(t, z, \delta)=0$. Таким образом, функция $\xi\left(\eta_{0}\right)(\cdot, \cdot, \cdot) \in \widetilde{K}\left([a, b] \times \mathbb{R}^{n} \times[0, \infty)\right)$. Лемма доказана.

Отметим, что аппроксимируюшее дифференциальное включение (1) с внешними возмушениями (включение (9)) определяется погрешностями вычисления значений отображения $F:[a, b] \times \mathbb{R}^{n} \rightarrow \operatorname{comp}\left[\mathbb{R}^{n}\right]$ (правой части включения (1)), которые задаются парой $(\widetilde{F}(\cdot, \cdot, \cdot), \xi(\cdot, \cdot, \cdot))$, и погрешшостями вычисления значений самого аппроксимирующего отображения $\widetilde{F}:[a, b] \times \mathbb{R}^{n} \times[0, \infty) \rightarrow \operatorname{comp}\left[\mathbb{R}^{n}\right]$. В то же время каждое решение $x:[a, b] \rightarrow \mathbb{R}^{n}$ включения (9) может также вычисляться с некоторой степеню точности, которую можно задать некоторой функцией $\eta_{0}(\cdot, \cdot, \cdot) \in \widetilde{K}\left([a, b] \times \mathbb{R}^{n} \times[0, \infty)\right)$. В связи с этим рассмотрим отображение $\widetilde{F}_{0}:[a, b] \times \mathbb{R}^{n} \times[0, \infty) \rightarrow \operatorname{comp}\left[\mathbb{R}^{n}\right]$, определенное равенством

$$
\widetilde{F}_{0}(t, x, \delta)=\overline{\widetilde{F}\left(t, B\left[x, \eta_{0}(t, x, \delta)\right], \delta\right)},
$$

где функция $\eta_{0}(\cdot, \cdot, \cdot) \in \widetilde{K}\left([a, b] \times \mathbb{R}^{n} \times[0, \infty)\right)$ в каждой точке $(t, x(t)) \in[a, b] \times \mathbb{R}^{n}$ при каждом фиксированном $\delta \in[0, \infty)$ задает погрешность вычисления значения решения $x:[a, b] \rightarrow \mathbb{R}^{n}$ в точке $t \in[a, b]$ дифференциального включения (9), причем эти погрешности могут быть неравномерны относительно фазовой переменной $x \in \mathbb{R}^{n}$. Далее, функцию $\eta_{0}(\cdot, \cdot, \cdot)$ будем называть радиусом внутренних возмущений аппроксимирующего отображсения $\widetilde{F}(\cdot, \cdot \cdot, \cdot)$ или просто радиусом внутренних возмущений.

Лемма 3. Пусть $\eta_{0}(\cdot, \cdot, \cdot) \in \widetilde{K}\left([a, b] \times \mathbb{R}^{n} \times[0, \infty)\right)$ u napa $(\widetilde{F}(\cdot, \cdot, \cdot), \xi(\cdot, \cdot, \cdot))$ аппроксимирует отображение $F(\cdot, \cdot)$. Тогда для отображсения $\widetilde{F}_{0}:[a, b] \times$ $\mathbb{R}^{n} \times[0, \infty) \rightarrow \operatorname{comp}\left[\mathbb{R}^{n}\right]$, заданного равенством $(29)$, при почти всех $t \in[a, b]$ и всех $(x, \delta) \in \mathbb{R}^{n} \times[0, \infty)$ справедливы соотношения

$$
\begin{gathered}
\left\|\widetilde{F}_{0}(t, x, \delta)\right\| \leqslant \sup _{z \in B\left[x, \eta_{0}(t, x, \delta)\right]}\|F(t, z)\|+\xi\left(\eta_{0}\right)(t, x, \delta), \\
h\left[F(t, x), \widetilde{F}_{0}(t, x, \delta)\right] \leqslant \xi\left(\eta_{0}\right)(t, x, \delta)+\varphi\left(\eta_{0}\right)(t, x, \delta),
\end{gathered}
$$


где функиия $\xi\left(\eta_{0}\right)(\cdot, \cdot, \cdot)$ определена равенством $(26)$, а $\varphi\left(\eta_{0}\right)(\cdot, \cdot, \cdot)$ - модуль непрерывности отображсения $F(\cdot, \cdot)$ относительно радиуса непрерывности $\eta_{0}(\cdot, \cdot, \cdot)$.

ДоказАТЕльство. Вначале докажем неравенство (30). Пусть $y \in \widetilde{F}_{0}(t, x, \delta)$, и пусть найдется такой $z \in B\left[x, \eta_{0}(t, x, \delta)\right]$, что $y \in \widetilde{F}(t, z, \delta)$. Так как для любого $p \in F(t, z)$ справедливо неравенство $|y| \leqslant|p|+|p-y|$, то

$$
\begin{aligned}
|y| & \leqslant\|F(t, z)\|+\rho[y, F(t, z)] \leqslant\|F(t, z)\|+h[\widetilde{F}(t, z, \delta), F(t, z)] \\
& \leqslant\|F(t, z)\|+\xi(t, z, \delta) \leqslant \sup _{z \in B\left[x, \eta_{0}(t, x, \delta)\right]}\|F(t, z)\|+\xi\left(\eta_{0}\right)(t, x, \delta) .
\end{aligned}
$$

Так как согласно неравенству (5) при почти всех $t \in[a, b]$ отображение $\widetilde{F}(\cdot, \cdot, \cdot)$ ограничено в шаре $B\left[x, \eta_{0}(t, x, \delta)\right]$, то из доказанной выше оценки вытекает неравенство (29).

Далее, докажем неравенство (31). Очевидно что при почти всех $t \in[a, b]$ и всех $(x, \delta) \in \mathbb{R}^{n} \times[0, \infty)$ справедливо соотношение $h^{+}\left[F(t, x) ; \widetilde{F}_{0}(t, x, \delta)\right] \leqslant \xi(t, x, \delta)$.

Тогда

Далее, оценим $h^{+}\left[\widetilde{F}_{0}(t, x, \delta) ; F(t, x)\right]$. Пусть $y \in \widetilde{F}(t, z, \delta)$ и $z \in B\left[x, \eta_{0}(t, x, \delta)\right]$.

$$
\begin{aligned}
\rho[y, F(t, x)] & \leqslant h^{+}[\widetilde{F}(t, z, \delta) ; F(t, x)] \leqslant h^{+}[\widetilde{F}(t, z, \delta) ; F(t, z)]+h^{+}[F(t, z) ; F(t, x)] \\
& \leqslant \xi(t, z, \delta)+\varphi\left(\eta_{0}\right)(t, x, \delta) \leqslant \xi\left(\eta_{0}\right)(t, x, \delta)+\varphi\left(\eta_{0}\right)(t, x, \delta) .
\end{aligned}
$$

Так как $\xi(\cdot, \cdot, \cdot) \leqslant \xi\left(\eta_{0}\right)(\cdot, \cdot, \cdot)+\varphi\left(\eta_{0}\right)(\cdot, \cdot, \cdot)$, то из полученных выше неравенств вытекает оценка (31). Лемма доказана.

ЗАмЕчанИЕ 5. Отметим, что из оценки (31) и лемм 1 и 2 следует, что отображение $\widetilde{F}_{0}:[a, b] \times \mathbb{R}^{n} \times[0, \infty) \rightarrow \operatorname{comp}\left[\mathbb{R}^{n}\right]$, заданное равенством $(29)$, аппроксимирует отображение $F:[a, b] \times \mathbb{R}^{n} \rightarrow \operatorname{comp}\left[\mathbb{R}^{n}\right]$ со степенњю аппроксимации $\xi(\cdot, \cdot, \cdot)=\xi\left(\eta_{0}\right)(\cdot, \cdot, \cdot)+\varphi\left(\eta_{0}\right)(\cdot, \cdot, \cdot)$.

Лемма 4. Пусть отображсения $\widetilde{F}:[a, b] \times \mathbb{R}^{n} \times[0, \infty) \rightarrow \operatorname{comp}\left[\mathbb{R}^{n}\right]$ u $\eta_{0}:[a, b] \times$ $\mathbb{R}^{n} \times[0, \infty) \rightarrow \mathbb{R}^{n}$ обладают свойством $\mathrm{B}$, с функииями $q:[a, b] \times[0, \infty) \rightarrow[0, \infty)$ $u \widetilde{q}:[a, b] \times[0, \infty) \rightarrow[0, \infty)$ соответственно, причем функиия $\widetilde{q}(\cdot, \cdot)$ ограничена при кажсдом ограниченном изменении второго аргумента. Тогда для отображсения $\widetilde{F}_{0}:[a, b] \times \mathbb{R}^{n} \times[0, \infty) \rightarrow \operatorname{comp}\left[\mathbb{R}^{n}\right]$, определенного равенством (29), при почти всех $t \in[a, b]$ и всех $x, y \in \mathbb{R}^{n}$ и $\delta \in[0, \infty)$ имеет место оиенка

$$
h\left[\widetilde{F}_{0}(t, x, \delta), \widetilde{F}_{0}(t, y, \delta)\right] \leqslant q(t, \delta)(1+\widetilde{q}(t, \delta))|x-y| .
$$

Действительно, согласно условию леммы 4 и леммам 2 и 4 из [14] при почти всех $t \in[a, b]$ и всех $x, y \in \mathbb{R}^{n}$ и $\delta \in[0, \infty)$ получаем неравенства

$$
\begin{aligned}
h\left[\widetilde{F}_{0}(t, x, \delta), \widetilde{F}_{0}(t, y, \delta)\right] & \leqslant q(t, \delta) h\left[B\left[x, \eta_{0}(t, x, \delta)\right], B\left[y, \eta_{0}(t, x, \delta)\right]\right] \\
& \leqslant q(t, \delta)(1+\widetilde{q}(t, \delta))|x-y|
\end{aligned}
$$

Поэтому оценка (32) справедлива. 
Пусть $\eta_{0}(\cdot, \cdot, \cdot) \in \widetilde{K}\left([a, b] \times \mathbb{R}^{n} \times[0, \infty)\right)$ и $\eta(\cdot, \cdot, \cdot) \in K\left([a, b] \times \mathbb{R}^{n} \times[0, \infty)\right)$. Рассмотрим отображение $Q_{\eta_{0}, \eta}:[a, b] \times \mathbb{R}^{n} \times[0, \infty) \rightarrow \operatorname{comp}\left[\mathbb{R}^{n}\right]$, определенное равенством

$$
Q_{\eta_{0}, \eta}(t, x, \delta)=\left(\widetilde{F}_{0}(t, x, \delta)\right)^{\eta(t, x, \delta)} .
$$

Согласно замечанию 5 для отображения $Q_{\eta_{0}, \eta}:[a, b] \times \mathbb{R}^{n} \times[0, \infty) \rightarrow \operatorname{comp}\left[\mathbb{R}^{n}\right]$ из неравенства (7) при почти всех $t \in[a, b]$ и всех $(x, \delta) \in \mathbb{R}^{n} \times[0, \infty)$ вытекает оценка

$$
h\left[F(t, x), Q_{\eta_{0}, \eta}(t, x, \delta)\right] \leqslant \xi\left(\eta_{0}\right)(t, x, \delta)+\varphi\left(\eta_{0}\right)(t, x, \delta)+\eta(t, x, \delta) .
$$

Поэтому для каждых функций $\eta_{0}(\cdot, \cdot, \cdot) \in \widetilde{K}\left([a, b] \times \mathbb{R}^{n} \times[0, \infty)\right)$ и $\eta(\cdot, \cdot, \cdot) \in$ $K\left([a, b] \times \mathbb{R}^{n} \times[0, \infty)\right)$ при почти всех $t \in[a, b]$ и всех $x \in \mathbb{R}^{n}$ справедливо равенство

$$
\lim _{\delta \rightarrow 0+0} h\left[F(t, x), Q_{\eta_{0}, \eta}(t, x, \delta)\right]=0 .
$$

Рассмотрим при каждом фиксированном $\delta \in[0, \infty)$ дифференциальное включение

$$
\dot{x}(t) \in Q_{\eta_{0}, \eta}(t, x(t), \delta), \quad t \in[a, b],
$$

где отображение $Q_{\eta_{0}, \eta}:[a, b] \times \mathbb{R}^{n} \times[0, \infty) \rightarrow \operatorname{comp}\left[\mathbb{R}^{n}\right]$ определено равенством (33). Дифференциальное включение (34) будем назьвать аппроксимирующим дифференциальное включение (1) с внутренними и внешними возмущениями.

Пусть $V \subset C^{n}[a, b]$. Обозначим через $H_{\eta_{0}(\delta), \eta(\delta)}(V)$ множество решений включения (34), принадлежащее заданному множеству $V$.

Из теорем 1-5 и лемм 2-4 вытекают следствия:

СЛЕДСТВИЕ 1. Пусть $V \subset C^{n}[a, b]$ - ограниченное замкнутое множество пространства $C^{n}[a, b]$, и пусть $\psi(\cdot, \cdot, \cdot) \in P\left([a, b] \times \mathbb{R}^{n} \times[0, \infty)\right), \eta_{0}(\cdot, \cdot, \cdot)$ $\in \widetilde{K}\left([a, b] \times \mathbb{R}^{n} \times[0, \infty)\right)$. Далее, пусть $(\widetilde{F}(\cdot, \cdot, \cdot), \xi(\cdot, \cdot, \cdot))$ aппроксимирует отображсние $F(\cdot, \cdot)$. Тогда для любой функиии $\eta(\cdot, \cdot, \cdot) \in K\left([a, b] \times \mathbb{R}^{n} \times\right.$ $[0, \infty))$, для которой существует такое число $\varepsilon>0$, что при почти всех $t \in[a, b]$, всех $x \in(U(V))^{\varepsilon}($ см. (13)) и $\delta \in[0, \infty)$ имеет место неравенство

$$
\xi\left(\eta_{0}\right)(t, x, \delta)+\varphi\left(\eta_{0}\right)(t, x, \delta)+\varphi(\psi)(t, x, \delta) \leqslant \eta(t, x, \delta)
$$

где функиия $\xi\left(\eta_{0}\right)(\cdot, \cdot, \cdot)$ определена равенством $(26), \varphi(\cdot)(\cdot, \cdot, \cdot)-$ модуль непрерывности отображения $F(\cdot, \cdot)$ относительно радиусов непрерывности $\eta_{0}(\cdot, \cdot, \cdot)$ и $\psi(\cdot, \cdot, \cdot)$, выполняется соотношение

$$
H_{\mathrm{co}}(V)=\bigcap_{\delta>0} \overline{H_{\eta_{0}(\delta), \eta(\delta)}\left(V^{\delta}\right)}
$$

где $\overline{H_{\eta_{0}(\delta), \eta(\delta)}\left(V^{\delta}\right)}$ - замыкание мнохества $H_{\eta_{0}(\delta), \eta(\delta)}\left(V^{\delta}\right)$ в пространстве $C^{n}[a, b], V^{\delta}-$ замкнутая в пространстве $C^{n}[a, b] \delta$-окрестность множества $V$. 
СлеДСТВИЕ 2. Пусть $V \subset C^{n}[a, b]$ - ограниченное замкнутое множество пространства $C^{n}[a, b]$ и $\eta_{0}(\cdot, \cdot, \cdot) \in \widetilde{K}\left([a, b] \times \mathbb{R}^{n} \times[0, \infty)\right)$. Далее, пусть пара $(\widetilde{F}(\cdot, \cdot, \cdot), \xi(\cdot, \cdot, \cdot))$ аппроксимирует отображение $F(\cdot, \cdot)$. Тогда для того чтобъ для любой функиии $\eta(\cdot, \cdot, \cdot) \in K\left([a, b] \times \mathbb{R}^{n} \times[0, \infty)\right)$ при почти всех $t \in[a, b]$ и всех $(x, \delta) \in \overline{U(V)} \times[0, \infty)($ см. (13)) удовлетворяющей соотночению

$$
\xi\left(\eta_{0}\right)(t, x, \delta)+\varphi\left(\eta_{0}\right)(t, x, \delta) \leqslant \eta(t, x, \delta),
$$

где функиия $\xi\left(\eta_{0}\right)(\cdot, \cdot, \cdot)$ определена равенством $(26), \varphi\left(\eta_{0}\right)(\cdot, \cdot, \cdot)$ - модуль непрерывности отображсения $F(\cdot, \cdot)$ относительно радиуса непрерывности $\eta_{0}(\cdot, \cdot, \cdot)$, имело место равенство

$$
\overline{H(V)}=\bigcap_{\delta>0} \overline{H_{\eta_{0}(\delta), \eta(\delta)}\left(V^{\delta}\right)}
$$

необходимо и достаточно, чтобы для включения (1) на мнохестве $V$ въполнялся приниип плотности.

Пусть $M \in \operatorname{comp}\left[\mathbb{R}^{n}\right]$, и пусть $\eta_{0}(\cdot, \cdot, \cdot), \eta(\cdot, \cdot, \cdot) \in K\left([a, b] \times \mathbb{R}^{n} \times[0, \infty)\right)$. Обозначим через $H_{\eta_{0}(\delta) \eta(\delta)}(M)$ множество решений задачи Коши включения $(34)$, начальные значения которых принадлежат множеству $M$.

СлеДСтвИЕ 3. Пусть $M \in \operatorname{comp}\left[\mathbb{R}^{n}\right]$, u nycmb napa $(\widetilde{F}(\cdot, \cdot, \cdot), \xi(\cdot, \cdot, \cdot))$ аппроксимирует отобрахсение $F(\cdot, \cdot)$. Далее, пусть отображсене $F(\cdot, \cdot)$ удовлетворяет условию Липшица, а отображения $\widetilde{F}(\cdot, \cdot, \cdot)$ и $\eta_{0}(\cdot, \cdot, \cdot)$ удовлетворяют лемме 4. Тогда для любой функиии $\eta(\cdot, \cdot, \cdot) \in K\left([a, b] \times \mathbb{R}^{n} \times\right.$ $[0, \infty))$ имеют место равенства

$$
\overline{H(M)}=\bigcap_{\delta>0} \overline{H_{\eta_{0}(\delta), \eta(\delta)}(M)}=\bigcap_{\delta>0} \overline{H_{\eta_{0}(\delta), \eta(\delta)}\left(M^{\delta}\right)}
$$

әде $\overline{H_{\eta_{0}(\delta), \eta(\delta)}(M)}, \overline{H_{\eta_{0}(\delta), \eta(\delta)}\left(M^{\delta}\right)}$ - замыкания в пространстве $C^{n}[a, b]$ соответствующих мнохсеств.

СлЕДСТВИЕ 4. Пусть $V \subset C^{n}[a, b]$ - ограниченное замкнутое множество пространства $C^{n}[a, b], \psi(\cdot, \cdot, \cdot) \in P\left([a, b] \times \mathbb{R}^{n} \times[0, \infty)\right)$. Далее, пусть пара $(\widetilde{F}(\cdot, \cdot, \cdot), \xi(\cdot, \cdot, \cdot))$ аппроксимирует отображение $F(\cdot, \cdot)$ вложением. Тогда для любой функции $\eta_{0}(\cdot, \cdot, \cdot) \in \widetilde{K}\left([a, b] \times \mathbb{R}^{n} \times[0, \infty)\right)$ и любой функции $\eta(\cdot, \cdot, \cdot) \in K\left([a, b] \times \mathbb{R}^{n} \times[0, \infty)\right)$, для которой существует такое число $\varepsilon>0$, что при почти всех $t \in[a, b]$ и всех $(x, \delta) \in(U(V))^{\varepsilon} \times[0, \infty)($ c.м. (13)) виполняется оченка

$$
\varphi(\psi)(t, x, \delta) \leqslant \eta(t, x, \delta)
$$

справедливо равенство (35).

СЛЕДСТВИЕ 5. Пусть $V \subset C^{n}[a, b]$ - ограниченное замкнутое множество пространства $C^{n}[a, b]$. Далее, пусть пара $(\widetilde{F}(\cdot, \cdot, \cdot), \xi(\cdot, \cdot, \cdot))$ аппроксимирует отображсение $F(\cdot, \cdot)$ вложением. Тогда для того чтобъ для любъх функиий $\eta_{0}(\cdot, \cdot, \cdot) \in \widetilde{K}\left([a, b] \times \mathbb{R}^{n} \times[0, \infty)\right)$ u $\eta(\cdot, \cdot, \cdot) \in K\left([a, b] \times \mathbb{R}^{n} \times[0, \infty)\right)$ имело место равенство (36), необходимо и достаточно, чтобы для включения (1) на множестве $V$ выполнялся принцип плотности. 
2.2. Аппроксимация вложением и с положительной оценкой снизу радиуса внутренних возмушений. Здесь предполагается, что радиус внутренних возмущений $\eta_{0}(\cdot, \cdot, \cdot) \in P\left([a, b] \times \mathbb{R}^{n} \times[0, \infty)\right)$, пара $(\widetilde{F}(\cdot, \cdot, \cdot), \xi(\cdot, \cdot, \cdot))$ аппроксимирует отображение $F(\cdot, \cdot)$ вложением, а аппроксимирующее отображение $\widetilde{F}:[a, b] \times \mathbb{R}^{n} \times[0, \infty) \rightarrow \operatorname{comp}\left[\mathbb{R}^{n}\right]$ обладает свойством: для любых $(x, \delta) \in$ $\mathbb{R}^{n} \times[0, \infty) \widetilde{F}(\cdot, x, \delta)$ измеримо; при почти всех $t \in[a, b]$ и всех $\delta \in[0, \infty) \widetilde{F}(t, \cdot, \delta)$ непрерывно (ниже это свойство для отображения $\widetilde{F}(\cdot, \cdot, \cdot)$ будем называть условием Каратеодори). В этом случае, как оказалось, следствие 4 можно уточнить.

Пусть $\eta_{0}(\cdot, \cdot, \cdot) \in P\left([a, b] \times \mathbb{R}^{n} \times[0, \infty)\right)$ и $\eta(\cdot, \cdot, \cdot) \in K\left([a, b] \times \mathbb{R}^{n} \times[0, \infty)\right)$. Определим отображения $\Phi:[a, b] \times \mathbb{R}^{n} \times[0, \infty) \rightarrow \operatorname{comp}\left[\mathbb{R}^{n}\right], \Phi_{\eta_{0} \eta}:[a, b] \times \mathbb{R}^{n} \times$ $[0, \infty) \rightarrow \operatorname{comp}\left[\mathbb{R}^{n}\right]$ равенствами

$$
\begin{aligned}
\Phi(t, x, \delta) & =\overline{\operatorname{ext}}(\operatorname{co} \widetilde{F}(t, x, \delta)), \\
\Phi_{\eta_{0} \eta}(t, x, \delta) & =\left(\Phi\left(t, B\left[x, \eta_{0}(t, x, \delta)\right], \delta\right)\right)^{\eta(t, x, \delta)},
\end{aligned}
$$

где $\overline{\operatorname{ext}}(\cdot)$ - замыкание множества крайних точек соответствуюшего множества. Для каждого фиксированного $\delta>0$ рассмотрим дифференциальное включение

$$
\dot{x}(t) \in \Phi_{\eta_{0} \eta}(t, x(t), \delta), \quad t \in[a, b],
$$

где отображение $\Phi_{\eta_{0} \eta}:[a, b] \times \mathbb{R}^{n} \times[0, \infty) \rightarrow \operatorname{comp}\left[\mathbb{R}^{n}\right]$ определено равенствами (37), (38). Дифференциальное включение (39) будем называть аппроксимирующим дифференииальное включение (1), определенное по крайним точкам значений аппроксимируюшего отображения $\widetilde{F}(\cdot, \cdot \cdot, \cdot)$, с внутренними и внешними возмущениями.

Пусть $\widetilde{H}_{\eta_{0}(\delta) \eta(\delta)}(V)$ - множество решений включения (39), принадлежащих множеству $V \subset C^{n}[a, b]$, при фиксированном $\delta>0$.

Отметим, что поскольку для любых $(t, x, \delta) \in[a, b] \times \mathbb{R}^{n} \times[0, \infty)$ выполняется включение $\Phi_{\eta_{0} \eta}(t, x, \delta) \subset Q_{\eta_{0} \eta}(t, x, \delta)$, где отображения $\Phi_{\eta_{0} \eta}(\cdot, \cdot, \cdot)$ и $Q_{\eta_{0} \eta}(\cdot, \cdot, \cdot)$ определены равенствами $(38)$ и $(33)$ соответственно, то

$$
\widetilde{H}_{\eta_{0}(\delta) \eta_{0}(\delta)}(V) \subset H_{\eta_{0}(\delta) \eta_{0}(\delta)}(V) .
$$

Рассмотрим также при каждом $\delta>0$ дифференциальные включения

$$
\begin{aligned}
& \dot{x}(t) \in \operatorname{co} \widetilde{F}(t, x(t), \delta), \quad t \in[a, b], \\
& \dot{x}(t) \in \Phi(t, x(t), \delta), \quad t \in[a, b] \text {. }
\end{aligned}
$$

Пусть $\mathscr{H}_{\text {со }}(V, \delta)$ - множество решений включения $(40)$, а $\widetilde{\mathscr{H}}_{\text {ext }}(V, \delta)$ - множество квазирешений включения (41), принадлежащих множеству $V \subset C^{n}[a, b]$.

Теорема 6. Пусть $V$ - замкнутое мнохсество пространства $C^{n}[a, b]$, $\eta_{0}(\cdot, \cdot, \cdot) \in P\left([a, b] \times \mathbb{R}^{n} \times[0, \infty)\right)$. Далее, nусть пара $(\widetilde{F}(\cdot, \cdot, \cdot), \xi(\cdot, \cdot, \cdot))$ аппроксимирует отображсение $F(\cdot, \cdot)$ вложсением и $\widetilde{F}(\cdot, \cdot, \cdot)$ удовлетворяет условиям Каратеодори. Тогда для любой функиии $\eta(\cdot, \cdot, \cdot) \in K\left([a, b] \times \mathbb{R}^{n} \times\right.$ $[0, \infty))$ справедливъ равенства

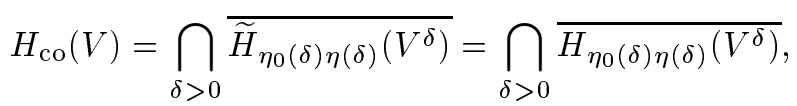

әде $\overline{\widetilde{H}_{\eta_{0}(\delta) \eta(\delta)}\left(V^{\delta}\right)}-$ замыкание множества $\widetilde{H}_{\eta_{0}(\delta) \eta(\delta)}\left(V^{\delta}\right)$ в пространстве $C^{n}[a, b]$. 
ДокАЗАтЕльство. Докажем первое равенство в (42). Вначале покажем, что

$$
H_{\mathrm{co}}(V) \subset \bigcap_{\delta>0} \overline{\widetilde{H}_{\eta_{0}(\delta) \eta(\delta)}\left(V^{\delta}\right)}
$$

Пусть $x \in H_{\text {со }}(V)$ и $\delta>0$. Так как $F(t, x) \subset \widetilde{F}(t, x, \delta)$, то со $F(t, x) \subset \operatorname{co~} \widetilde{F}(t, x, \delta)$ и, следовательно, $H_{\text {со }}(V) \subset \mathscr{H}_{\text {со }}(V, \delta)$. Поскольку $\mathscr{H}_{\text {со }}(V, \delta) \subset \widetilde{\mathscr{H}}_{\text {ext }}\left(V^{\delta}, \delta\right)($ см. $[6],[7])$, то $x(\cdot) \in \widetilde{\mathscr{H}}_{\text {ext }}\left(V^{\delta}, \delta\right)$, и поэтому найдется такая последовательность абсолютно непрерывных функций $x_{i}:[a, b] \rightarrow \mathbb{R}^{n}, i=1,2, \ldots$, что $x_{i} \rightarrow x$ в $C^{n}[a, b]$ при $i \rightarrow \infty$ и для любого $i=1,2, \ldots$ при почти всех $t \in[a, b]$ выполняется включение

$$
\dot{x}_{i}(t) \in \Phi(t, x(t), \delta),
$$

где отображение $\Phi(\cdot, \cdot, \cdot)$ определено равенством $(37)$. Так как $\eta_{0}(\cdot, \cdot, \cdot) \in$ $P\left([a, b] \times \mathbb{R}^{n} \times[0, \infty)\right)$, то найдется такой номер $I$, что при всех $i \geqslant I$ и при почти всех $t \in[a, b]$ выполняется включение $x(t) \in B\left[x_{i}(t), \eta_{0}\left(t, x_{i}(t), \delta\right)\right]$. Поэтому из включения (44) при всех $i \geqslant I$ и при почти всех $t \in[a, b]$ имеют место соотношения

$$
\dot{x}_{i}(t) \in \Phi\left(t, B\left[x_{i}(t), \eta_{0}\left(t, x_{i}(t), \delta\right)\right], \delta\right) \subset \Phi_{\eta_{0} \eta}\left(t, x_{i}(t), \delta\right)
$$

Следовательно, при всех $i \geqslant I x_{i} \in \widetilde{H}_{\eta_{0}(\delta) \eta(\delta)}\left(V^{\delta}\right)$ и поэтому $x \in \overline{H_{\eta_{0}(\delta) \eta(\delta)}\left(V^{\delta}\right)}$. Таким образом, вложение (43) доказано.

Вложение

$$
\bigcap_{\delta>0} \overline{\widetilde{H}_{\eta_{0}(\delta) \eta(\delta)}\left(V^{\delta}\right)} \subset H_{\mathrm{co}}(V)
$$

доказывается аналогично включению (17) в теореме 1. Второе равенство (42) доказывается аналогично. Теорема доказана.

ЗАмечАнИЕ 6 . Отметим, что теоремы 1-6 и следствия $1-5$ обобщают и уточняют результаты работ [14]-[20].

\section{Список литературы}

1. Филиппов $A . \Phi$. Дифференциальные уравнения с разрывной правой частью. М.: Наука, 1985.

2. Wazewski T. Sur une generalisation de la notion des solutions d'une equation au contingent // Bull. Acad. Pol. Sci. Ser. Sci. Math. Astron. Phys. 1962. V. 10. № 1. P. 11-15.

3. Натансон И. Т. Теория функций вещественной переменной. М.: Наука, 1974.

4. Иоффе А. Д., Тихомиров В. М. Теория экстремальных задач. М.: Наука, 1974.

5. Turowicz A. Remarque sur la definition des quasitrajectoires d'un system de commande nonlineaire // Bull. Acad. Pol. Sci. Ser. Sci. Math. Astron. Phys. 1963. V. 11. №6. P. 367-368.

6. Булгаков А.И. Интегральные включения с невыпуклыми образами и их приложения к краевым задачам дифференциальных включений // Матем. сб. 1992. Т. 183. № 10. C. $63-86$.

7. Булгаков А.И., Ткач Л.И. Возмущение вьпуклозначного оператора многозначньм отображением типа Гаммерштейна с невьпукльми образами и краевые задачи для функционально-дифференциальных включений // Матем. сб. 1998. Т. 189. №6. С. 3-32.

8. Plis A. Trajectories and quasitrajectories of an orientor field // Bull. Acad. Pol. Sci. Ser. Sci. Math. Astron. Phys. 1963. V. 11. № 6. P. 369-370. 
9. Филиппов $A . \Phi$. Классические решения дифференциальных уравнений с многозначной правой частью // Вестн. МГУ. Сер.1. Матем. мех. 1967. № 3. С. 16-26.

10. Благодатских В.И., Филиппов А. Ф. Дифференциальные включения и оптимальное управление // Труды МИАН. 1985. Т. 169. С. 194-252.

11. Ирисов A.E., Тонков Е.Л. О замькании множества периодических решений дифференциального включения // Дифференциальные и интегральные уравнения. Горький: Изд-во ГГУ, 1983. С. 32-38.

12. Булгаков А. И. Непрерьвные ветви многозначных отображений и интегральные включения с невьпукльми образами и их приложения. I,II,III // Дифференц. уравнения. 1992. T. 28. №3. C. 371-379; № 4. C. 566-571; № 5. C. 739-746.

13. Pianigiani $G$. On the fundamental theory of multivalued differential equations // Differential Equations. 1977. V. 25. № 1. P. 30-38.

14. Булгаков А.И., Ефремов А.А., Панасенко Е.А. Обькновенные дифференциальные включения с внутренними и внешними возмущениями // Дифференц. уравнения. 2000. T. 36. № 12. C. 1587-1598.

15. Булгаков А.И. Асимптотическое представление множеств $\delta$-решений дифференциального включения // Матем. заметки. 1999. Т. 65. № 5. С. 775-778.

16. Булгаков А.И., Ефремов A. А., Панасенко E. А. К вопросу устойчивости дифференциальных включений // Вестн. Тамб.ГУ. Сер. естеств. и технич. науки. 1999. Т. 4. № 4. C. $461-469$.

17. Булгаков А.И., Ткач Л.И. Возмущение однозначного оператора многозначньм отображением типа Гаммерштейна с невьпукльми образами // Изв. вузов. Сер. матем. 1999. T. 442. № 3. C. 3-16.

18. Булгаков А.И., Скоморохов В.В. Дифференциальные включения с внешними возмущениями, радиус которых зависит от фазовой переменной // Вестн. Тамб. ГУ. Сер. естеств. и технич. науки. 2000. Т. 5. № 4. С. 429-430.

19. Ефремов А. А., Панасенко Е. А. Устойчивость периодических и двухточечных краевых задач относительно внешних возмущений // Вестн. Тамб. ГУ. Сер. естеств. и технич. науки. 2000. Т. 5. № 4. С. 446-447.

20. Hermes $H$. The generalised differential equation $\dot{x}(t) \in R(t, x) / /$ Adv. Math. 1970. V. 4 . № 2. P. 149-169.

Тамбовский государственнњй университет им. Г. Р. Державина;

Поступила в редакцию

Тамбовский государственный технический университет

31.01.2001

E-mail: aib@tsu.tmb.ru, uua@hmd.nnn.tstu.ru 